\title{
Changes in regional body fat, lean body mass and body shape in trans persons using cross- sex hormonal therapy: results from a multicenter prospective study
}

\author{
M Klaver', C J M de Blok', C M Wiepjes', N M Nota', M J H J Dekker', R de Mutsert², T Schreiner³, A D Fisher', \\ $\mathbf{G}^{\mathbf{T}}$ 'Sjoen $^{\mathbf{5}}$ and $\mathbf{M}$ den Heijer ${ }^{1}$
}

${ }^{1}$ Department of Endocrinology and Center of Expertise on Gender Dysphoria, VU University Medical Center, Amsterdam, the Netherlands, ${ }^{2}$ Department of Clinical Epidemiology, Leiden University and Medical Center, Leiden, the Netherlands, ${ }^{3}$ Department of Endocrinology, Oslo University Hospital, Oslo, Norway, ${ }^{4}$ Sexual Medicine and Andrology Unit, Department of Experimental, Clinical and Biomedical Sciences, University of Florence, Florence, Italy, and ${ }^{5}$ Department of Endocrinology \& Center for Sexuology and Gender, Ghent University Hospital, Ghent, Belgium

Correspondence should be addressed to $M$ den Heijer Email m.denheijer@vumc.nl

\begin{abstract}
Objective: Cross-sex hormonal therapy (CHT) in trans persons affects their total body fat and total lean body mass. However, it is unknown how separate body regions are affected and whether these changes alter body shape. Therefore, the aim of this study was to determine the effects on body fat and lean body mass in separate body regions and on body shape after one year of $\mathrm{CHT}$.

Design and methods: In a multicenter prospective study at university hospitals, 179 male-to-female gender dysphoric persons, referred to as transwomen, and 162 female-to-male gender dysphoric persons, referred to as transmen, were included. All underwent whole-body dual-energy $\mathrm{X}$-ray absorptiometry and anthropometric measurements before and after one year of $\mathrm{CHT}$.

Results: In transwomen, increases in body fat ranged from $+18 \%(95 \% \mathrm{Cl}: 13 \% ; 23 \%)$ in the android region to $+42 \%$ (95\% Cl: $37 \% ; 46 \%)$ in the leg region and $+34 \%(95 \% \mathrm{Cl}: 29 \% ; 38 \%)$ in the gynoid region. In transmen, changes in body fat ranged from $-16 \%(95 \% \mathrm{Cl}:-19 ;-14 \%)$ in the leg region and $-14 \%$ in the gynoid region ( $95 \% \mathrm{Cl}$ : $-16 \% ;-12)$ to no change in the android region $(+1 \%, 95 \% \mathrm{Cl}:-3 \% ; 5 \%)$. Waist-to-hip ratio (WHR) decreased in transwomen $(-0.03,95 \% \mathrm{Cl}:-0.04 ;-0.02)$ mainly due to an increase in hip circumference $(+3.2 \mathrm{~cm}, 95 \% \mathrm{Cl}: 2.3 ; 4.0)$. Transmen have a decrease in hip circumference $(-1.9 \mathrm{~cm}, 95 \% \mathrm{Cl}:-3.1 ;-0.7)$ resulting in an increase in WHR $(+0.01$, $95 \% \mathrm{Cl}: 0.00 ; 0.02)$.

Conclusions: CHT causes a more feminine body fat distribution and a lower WHR in transwomen and a more masculine body fat distribution with a lower hip circumference in transmen.
\end{abstract}

\section{Introduction}

Endogenous sex steroids such as testosterone and estradiol play important roles in the accumulation and distribution of body fat and lean body mass and thus in the
C) 2018 European Society of Endocrinology Printed in Great Britain
European Journal of

Endocrinology

(2018) 178, 163-171 feminization and masculinization of body composition (1). This is illustrated in puberty, when, due to increasing estrogens, girls develop more total body fat and mainly 
store fat in the gluteal and femoral regions (so-called 'pear' or gynoid body shape), whereas boys obtain more muscle mass and store body fat predominantly in the abdominal area ('apple' or android body shape), presumably due to higher testosterone levels (1).

Persons with gender dysphoria are treated with exogenous sex steroids or 'cross-sex hormonal therapy' (CHT) to obtain the physical characteristics of the desired sex, including a feminine or masculine body fat distribution, musculature and body shape. Male-to-female gender dysphoric persons, referred to as transwomen, are treated with estradiol preparations in combination with an anti-androgen, whereas female-to-male gender dysphoric persons, referred to as transmen, receive testosterone preparations. In a recent meta-analysis, we showed that, during the first year of CHT, total body fat increases and total lean body mass decreases in transwomen, while the opposite occurs in transmen (2). However, little is known about whether the extent of changes in body fat and lean body mass differs between body parts and whether the preferred site of fat deposition changes during $\operatorname{CHT}(3,4)$.

Both untreated transwomen and transmen reported to be unsatisfied with their figure, waist region and hip region before hormonal therapy (5). Some transwomen even inject silicone liquids into their femoral region in order to obtain broader hips, showing the great desire for a feminine body shape phenotype (6). Changes in body composition and body fat distribution might also affect body shape. So far, only few small studies reported on these effects in the first year of treatment and showed inconsistent effects on waist and hip circumferences and waist-hip ratio (WHR) $(4,7,8)$.

Therefore, the aim of our study was to determine the effects of CHT on body fat and lean body mass in separate body regions and the effects on measures of body shape as waist and hip circumferences and waist-hip ratio (WHR). In addition, we evaluated whether factors such as medication type, serum sex hormone levels, BMI at start and age affect the changes in aforementioned measures.

\section{Methods}

\section{Study population and study design}

This study is embedded in the European Network for the Investigation of Gender Incongruence (ENIGI) project, a prospective observational study conducted in four collaborating gender clinics in Amsterdam, Ghent,
Oslo and Florence. The full protocol has been published elsewhere (9), and the study is registered at https:// clinicaltrials.gov/ct2/show/NCT01072825. All persons of 18 years and older diagnosed with GD $(10,11)$ and starting CHT between 2010 and April 2016 were eligible for participation in the study. Persons were not eligible when they started in a different treatment protocol (e.g. the use of spironolactone) or in case of previous crosssex hormone use, insufficient knowledge of the spoken language or psychological vulnerability. Participants visited the outpatient clinic every three months during the first year of CHT for clinical data collection (e.g. the use of other medication), the measurement of anthropometrics and laboratory tests to examine whether appropriate sex hormone levels were reached.

For the present analyses, participants were included if they completed one year of CHT and had undergone whole-body dual-energy X-ray absorptiometry (DXA) at the start and after one year of CHT. In participating centers, different type of DXA scanners were used (Amsterdam and Ghent: Hologic Discovery A, Oslo: Lunar, Florence: Hologic Delphi). Because the use of different types of DXA scanners results in non-comparable body composition data, only participants from Amsterdam and Ghent were selected for these analyses. Persons were excluded if the baseline DXA was made $<90$ days before the start of CHT or $>31$ days after the start of CHT. In addition, persons were excluded if the follow-up DXA was obtained $<10$ months or $>14$ months after the start of CHT. These inclusions and exclusions resulted in 341 participants for the present analyses. The study population of $n=341$ is larger than any study before on the relation of cross-sex hormonal treatment and the effects on body composition and body shape. We calculated that, for example, in the group of 179 transwomen, a mean difference in, for example, body fat of $5.6 \%$ or higher can be detected, with a power of $80 \%$ and alpha $=0.05$. The participant inclusion flow chart is shown in Fig. 1.

The Ethics Committee of Ghent University Hospital, Belgium approved the overall study protocol. The other participating centers also obtained approval of their local ethical committees. Informed consent was obtained according to the institutional guidelines.

\section{Treatment protocol}

Transwomen were treated with cyproterone acetate (CPA) $50 \mathrm{mg} /$ day in combination with oral estradiol valerate $4 \mathrm{mg} /$ day or a transdermal estradiol patch $100 \mu \mathrm{g} / 24 \mathrm{~h}$ twice a week. The latter was recommended if persons 


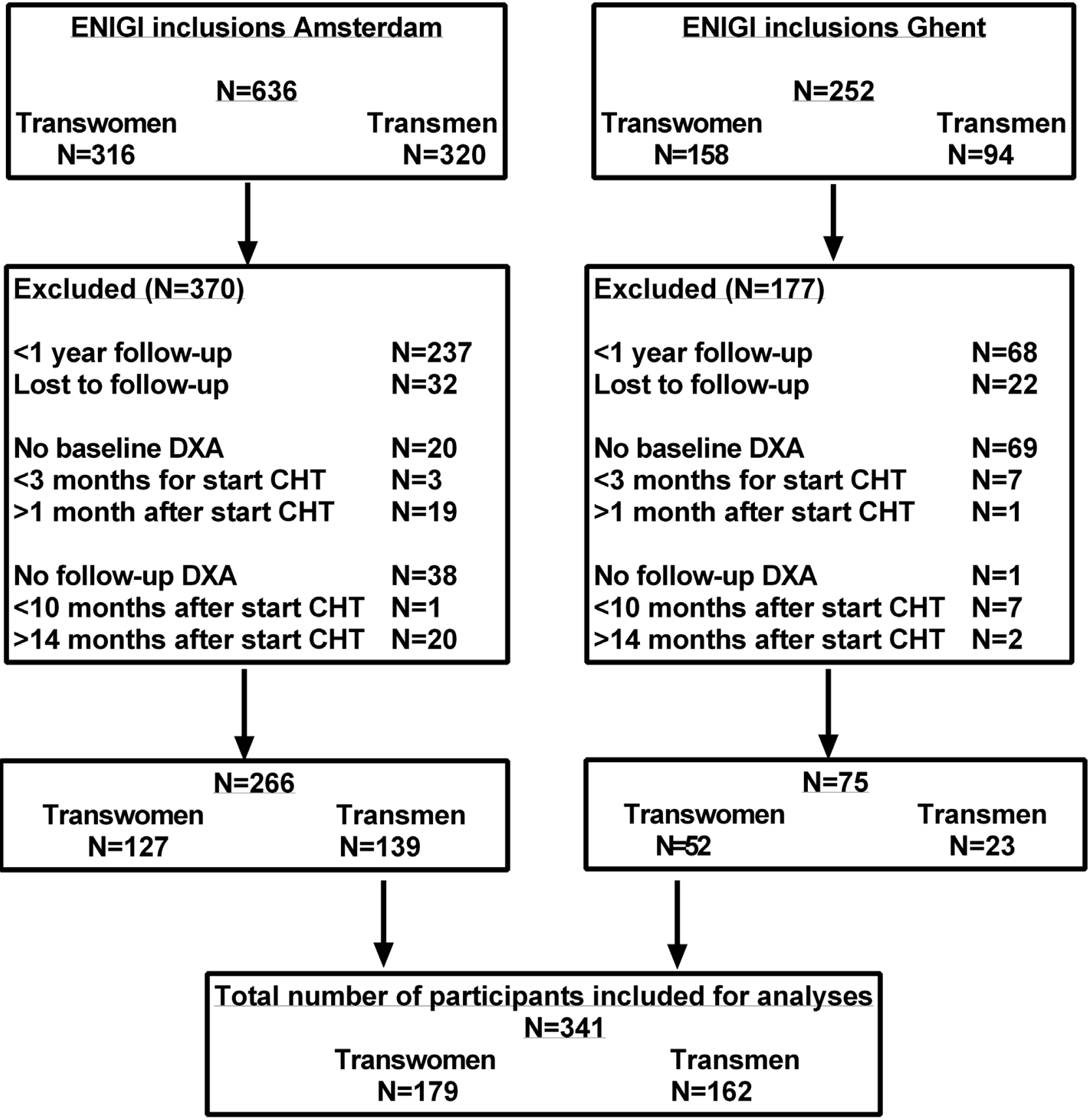

Figure 1

Flowchart of the participant inclusion. CHT, cross-sex hormonal therapy; DXA, dual-energy X-ray absorptiometry.

were above 40 years old or if they had a history of cardiovascular disease, hormone-sensitive malignancies or thromboembolic events. Transmen received testosterone

(T) gel $50 \mathrm{mg} /$ day, $\mathrm{T}$ undecanoate $1000 \mathrm{mg}$ intramuscular (i.m.) once per 12 weeks or T esters $250 \mathrm{mg}$ i.m. once per 2 weeks.
Anthropometrics

Body weight was measured in light indoor clothing without shoes. Waist circumference, defined as the smallest abdominal circumference, and hip circumference, measured at the level of the trochanter major, were 
Table 1 Baseline characteristics of the 179 transwomen and 162 transmen included in Amsterdam and Ghent between 2010 and 2016. Data are presented as number, percentage, mean (s.D.) or median (minimum and maximum value).

\begin{tabular}{l}
\hline \\
\hline Total number of participants $(n)$ \\
VU University Medical Center Amsterdam $(n)$ \\
Ghent University Hospital $(n)$ \\
Age at baseline (years) \\
Current smoking at baseline $(\%)$ \\
Body weight $(\mathrm{kg})$ \\
BMI $\left(\mathrm{kg} / \mathrm{m}^{2}\right)$ \\
Waist circumference $(\mathrm{cm})$ \\
Hip circumference $(\mathrm{cm})$ \\
WHR \\
Body fat \\
Total body $(\mathrm{kg})$ \\
Arm region $(\mathrm{kg})$ \\
Leg region $(\mathrm{kg})$ \\
Trunk region $(\mathrm{kg})$ \\
Android region $(\mathrm{kg})$ \\
Gynoid region $(\mathrm{kg})$ \\
Lean body mass \\
Total body $(\mathrm{kg})$ \\
Arm region $(\mathrm{kg})$ \\
Leg region $(\mathrm{kg})$ \\
Trunk region $(\mathrm{kg})$ \\
Android region $(\mathrm{kg})$ \\
Gynoid region $(\mathrm{kg})$ \\
\hline
\end{tabular}

\begin{tabular}{c} 
Transwomen \\
\hline 179 \\
127 \\
52 \\
$29(18-66)$ \\
25 \\
$74.5 \pm 12.7$ \\
$23.3 \pm 3.8$ \\
$82.6 \pm 11.1$ \\
$95.6 \pm 8.3$ \\
$0.86 \pm 0.08$ \\
$17.6 \pm 5.8$ \\
$1.0 \pm 0.3$ \\
$3.1 \pm 0.9$ \\
$7.9 \pm 3.1$ \\
$1.3 \pm 0.5$ \\
$3.0 \pm 0.9$ \\
\\
$57.2 \pm 8.3$ \\
$3.4 \pm 0.5$ \\
$9.7 \pm 1.4$ \\
$26.9 \pm 3.9$ \\
$3.9 \pm 0.7$ \\
$8.6 \pm 1.3$
\end{tabular}

determined with a tape measure. These measures were used to calculate the WHR.

\section{Body composition by DXA}

Body fat, lean body mass and total mass of the whole body and specific regions as the arm region, leg region, trunk region, android region and gynoid region were measured using DXA. The same type of DXA scanner was used in both centers (Hologic Discovery A, Hologic Inc., Bedford, MA, USA). All scans were analyzed using software, version 13.5.3. The separate body regions were defined using the software provided by Hologic (Supplementary Fig. 1, see section on supplementary data given at the end of this article) (12). The main outcome measures for regional body composition were the percentage change in body fat and lean body mass in the arm region, leg region, trunk region, android region and gynoid region.

\section{Blood sampling and sex hormone concentrations}

Serum estradiol (pmol/L) and testosterone $(\mathrm{nmol} / \mathrm{L})$ levels were determined at baseline and after 3 and 12 months of CHT. In Supplementary Tables 2 and 3, the type of assays used in both centers is described with their

\section{Statistical analyses}

inter-assay coefficients of variation and lower limits of quantification.

\begin{tabular}{c}
\hline Transmen \\
162 \\
139 \\
23 \\
$24(18-58)$ \\
28 \\
$70.3 \pm 14.3$ \\
$25.0 \pm 4.6$ \\
$80.1 \pm 12.3$ \\
$100.6 \pm 10.7$ \\
$0.80 \pm 0.08$ \\
$24.1 \pm 7.7$ \\
$1.4 \pm 0.5$ \\
$5.1 \pm 1.5$ \\
$9.9 \pm 3.8$ \\
$1.7 \pm 0.7$ \\
$4.7 \pm 1.2$ \\
$46.9 \pm 8.1$ \\
$2.5 \pm 0.4$ \\
$7.8 \pm 1.4$ \\
$22.6 \pm 3.6$ \\
$3.3 \pm 0.7$ \\
$7.4 \pm 1.3$ \\
\end{tabular}

Baseline characteristics were expressed as numbers, percentages, means with an S.D. in case of a normal distribution or medians with the minimum and maximum value in case of a non-normal distribution.

All outcome variables were normally distributed and therefore no log-transformation had to be performed before analyses. For the regional body composition outcomes, we calculated a mean relative increase of body fat or lean body mass by dividing the change in the outcome measure by the value at baseline multiplied by 100 . This was first done per participant, after which a mean change with a 95\% confidence interval was calculated. For body shape outcome measures (waist, hip and WHR), a mean change with a $95 \%$ confidence interval and a $P$ value was calculated with a Student's $t$-test. After these analyses on the crude changes, we performed linear regression to adjust these changes for age and BMI at start.

Next, we performed stratified linear regression analyses to examine whether subgroups of type of hormonal treatment, sex hormone levels, BMI at start, age or the 
use of other medication that affects body composition (antidepressants, antipsychotics, corticosteroids or antiepileptic drugs) influenced the changes in outcome measures.

Subgroups of type of hormonal treatment were $\mathrm{T}$ esters, T gel and T undecanoate for transmen. Subgroups in transwomen were oral estradiol and transdermal estradiol. In these analyses, only transwomen and transmen using the same type of treatment during the whole year were included. Analyses on sex hormone levels were performed in quartiles of $Z$-scores, which is described in more detail below. BMI at start was analyzed in the categories BMI $<20$, BMI 20-25, BMI 25.1-30 and BMI $>30 \mathrm{~kg} / \mathrm{m}^{2}$, and the categories for age were defined based on quartiles, resulting in the following categories: $<22,22-26,27-38$ and $>38$ years. Analyses on the use of other medication were performed in two subgroups (yes or no). Analyses were performed both crude and adjusted for age and BMI at start. Analyses on BMI at start were only adjusted for age, and analyses on age were only adjusted for BMI at start. For all analyses, persons with missing data were excluded.

Because different biochemical assays were used in Amsterdam and Ghent, the analyses on the effect of sex hormone levels on outcome measures were performed using $Z$-scores. A mean serum estradiol or testosterone level was calculated for each person by summing up the levels determined at the 3-month and 12-month visit and divided by 2. Mean (s.D.) estradiol levels in transwomen were 251 (138) pmol/L in Amsterdam and 268 (162) pmol/L in Ghent. Mean (s.D.) testosterone levels in transmen were $31(18) \mathrm{nmol} / \mathrm{L}$ in Amsterdam and 18 (7) $\mathrm{nmol} / \mathrm{L}$ in Ghent. These mean levels were standardized to a mean of zero and a S.D. of one, where after individual $Z$-scores could be calculated. Subsequently, the $Z$-scores were analyzed in categories based on quartiles, resulting in the following categories ( $<-0.7,-0.7$ to $0,0-0.7,>0.7)$ for both transwomen and transmen. Statistical analyses were performed using STATA, version 13.1 (StataCorp).

\section{Results}

In total, 179 transwomen and 162 transmen were included with a mean (s.D.) follow-up time of 377 (22) and 380 (22) days, respectively. The baseline characteristics of the study population are shown in Table 1. During the first year of CHT, in transwomen, body weight $(+3 \%$; 95\% CI: $2 \% ; 5 \%, P<0.001)$ and total body fat $(+28 \%$; 95\% CI: $24 \% ; 32 \%, P<0.001)$ increased, whereas total LBM decreased with $-3 \%$ (95\% CI: $-4 \% ;-2 \%, P<0.001)$. Transmen gained $+3 \%(95 \%$ CI: $2 \% ; 4 \%, P<0.001)$ in body
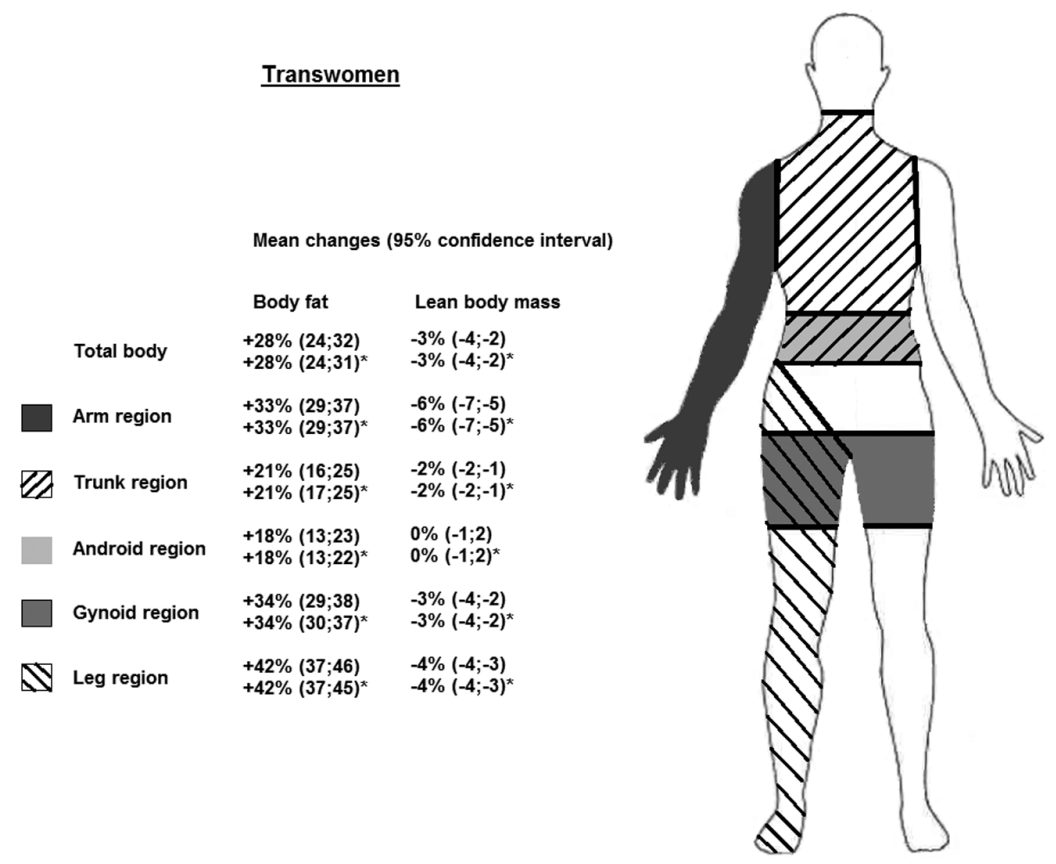

Transmen

$+42 \%(37 ; 45)^{*} \quad-4 \%(-4 ;-3)^{*}$

\section{Figure 2}

Percentage changes in total and regional body fat and lean body mass in 179 transwomen and 162 transmen after one year of cross-sex hormonal therapy. Effect estimates did not change after adjustment for body mass index at start and age. 
Transwomen

Mean change ( $95 \%$ confidence interval)

Waist: $\quad-0.6 \mathrm{~cm}(-1.7 ; 0.4)$ $-0.7 \mathrm{~cm}(-1.7 ; 0.3)$ *

Hip: $\quad+3.2 \mathrm{~cm}(2.3 ; 4.0)$ $+3.2 \mathrm{~cm}(2.3 ; 4.0)$ *

WHR: $\quad-0.03(-0.04 ;-0.02)$ $-0.03(-0.04 ;-0.02)$

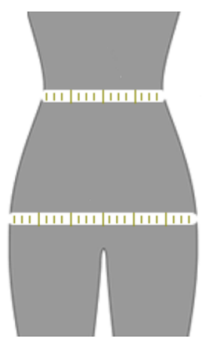

\section{Figure 3}

Changes in waist circumference, hip circumference and WHR in 179 transwomen and 164 transmen after one year of cross-sex hormonal therapy. *After adjustment for body mass index at start and age. weight with a decrease in total body fat of $-9 \%$ (95\% CI: $-12 \% ;-7 \%, P<0.001)$ and an increase in total LBM of $+10 \%$ (95\% CI: 9\%;11\%, $P<0.001)$.

\section{Changes in regional body fat, regional lean body mass and WHR in transwomen}

In transwomen, regional changes in body fat ranged from $+18 \%$ (95\% CI: 13\%;23\%, $P<0.001)$ in the android region to $+42 \%(95 \% \mathrm{CI}: 37 \% ; 46 \%, P<0.001)$ in the leg region.
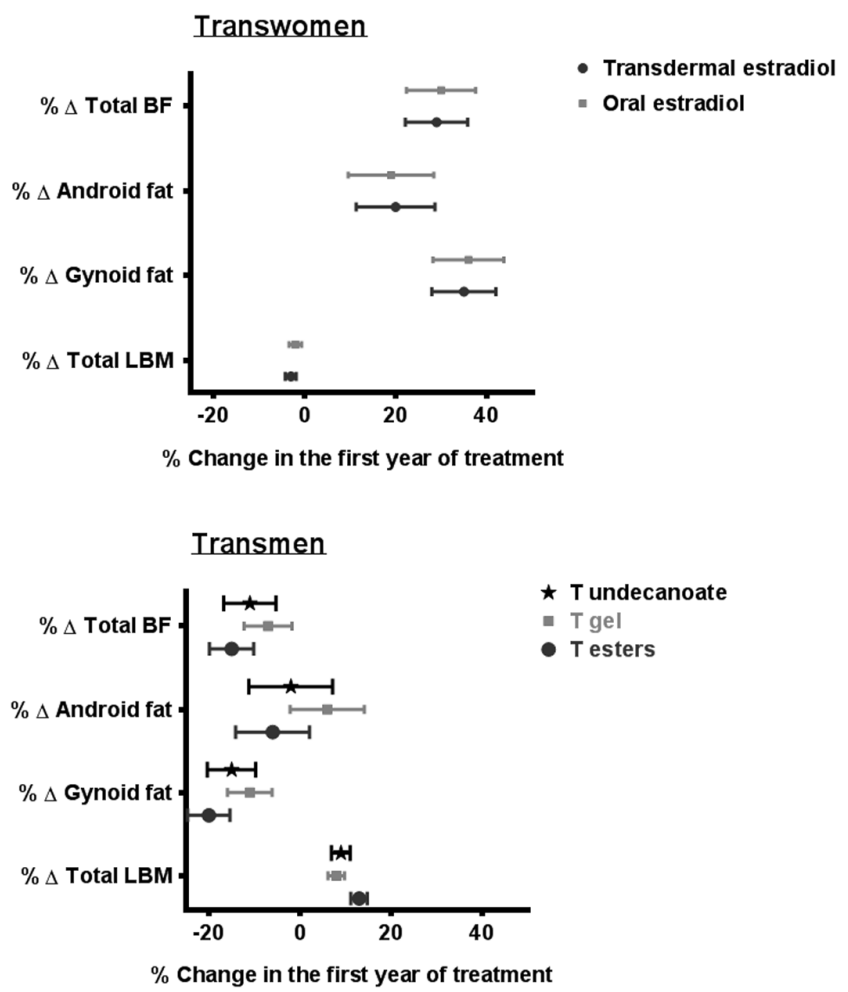

\section{Figure 4}

Percentage changes in body fat outcomes and total lean body mass per medication type in transwomen and transmen. T, testosterone. Data are presented as means $+95 \%$ confidence intervals.
Body fat in the gynoid region increased with $+34 \%(95 \%$ CI: 29\%;38\%, $P<0.001)$. Regional changes in LBM ranged from $0 \%(95 \% \mathrm{CI}:-1 \% ; 2 \%, P=0.61)$ in the android region to $-6 \%(95 \% \mathrm{CI}:-7 \% ;-5 \%, P<0.001)$ in the arm region (Fig. 2). Transwomen changed $-0.7 \mathrm{~cm}(95 \% \mathrm{CI}:-1.7 ; 0.3$, $P=0.23)$ in waist circumference and $+3.2 \mathrm{~cm}(95 \% \mathrm{CI}$ : 2.3;4.0, $P<0.001)$ in hip circumference after adjustment for age and BMI at start, resulting in a decrease in WHR (-0.03, 95\% CI: $-0.04 ;-0.02, P<0.001)$ (Fig. 3).

\section{Changes in regional body fat, regional LBM and WHR} in transmen

While transmen lost most body fat in the leg region $(-16 \%, 95 \% \mathrm{CI}:-19 \% ;-14 \%, P<0.001)$ and the gynoid region $(-14 \%, 95 \% \mathrm{CI}:-16 \% ;-12 \%, P<0.001)$, body fat in the android region $(+1 \%, 95 \% \mathrm{CI}:-3 \% ; 5 \%, P=0.63)$ did not change. LBM increased in all body parts, ranging from $+9 \%(95 \% \mathrm{CI}: 8 \% ; 10 \%, P<0.001)$ in the trunk to $+19 \%$ (95\% CI: 18\%;21\%, $P<0.001)$ in the arm (Fig. 2). Transmen changed $-0.3 \mathrm{~cm}(95 \% \mathrm{CI}:-1.6 ; 0.9, P=0.53)$ in waist circumference and $-1.9 \mathrm{~cm}(95 \% \mathrm{CI}:-3.1 ;-0.7$, $P<0.002)$ in hip circumference after adjustment for age and BMI at start and thus increased in WHR (+0.01, 95\% CI: 0.00;0.02, $P=0.03$ ) (Fig. 3).

\section{Type of cross-sex hormonal treatment}

Sixty-five transwomen using oral estrogens, 49 transwomen using transdermal estrogens, 41 transmen using $\mathrm{T}$ esters, 34 transmen using $\mathrm{T}$ gel and 30 transmen using $\mathrm{T}$ undecanoate were included. Crude analyses in transwomen showed a trend toward a larger change in some body fat outcomes in persons using oral estradiol compared with persons using transdermal estradiol, but after adjustment for BMI at the start and age, these differences disappeared (Supplementary Table 1). Transmen using $\mathrm{T}$ esters tended to experience larger changes than transmen using $\mathrm{T}$ gel or $\mathrm{T}$ undecanoate in 
Table 2 Percentage body fat or lean body mass in a specific body region before and after one year of cross-sex hormonal therapy in 179 transwomen and 162 transmen with reference values of the desired sex. Data are presented as percentages with S.E.

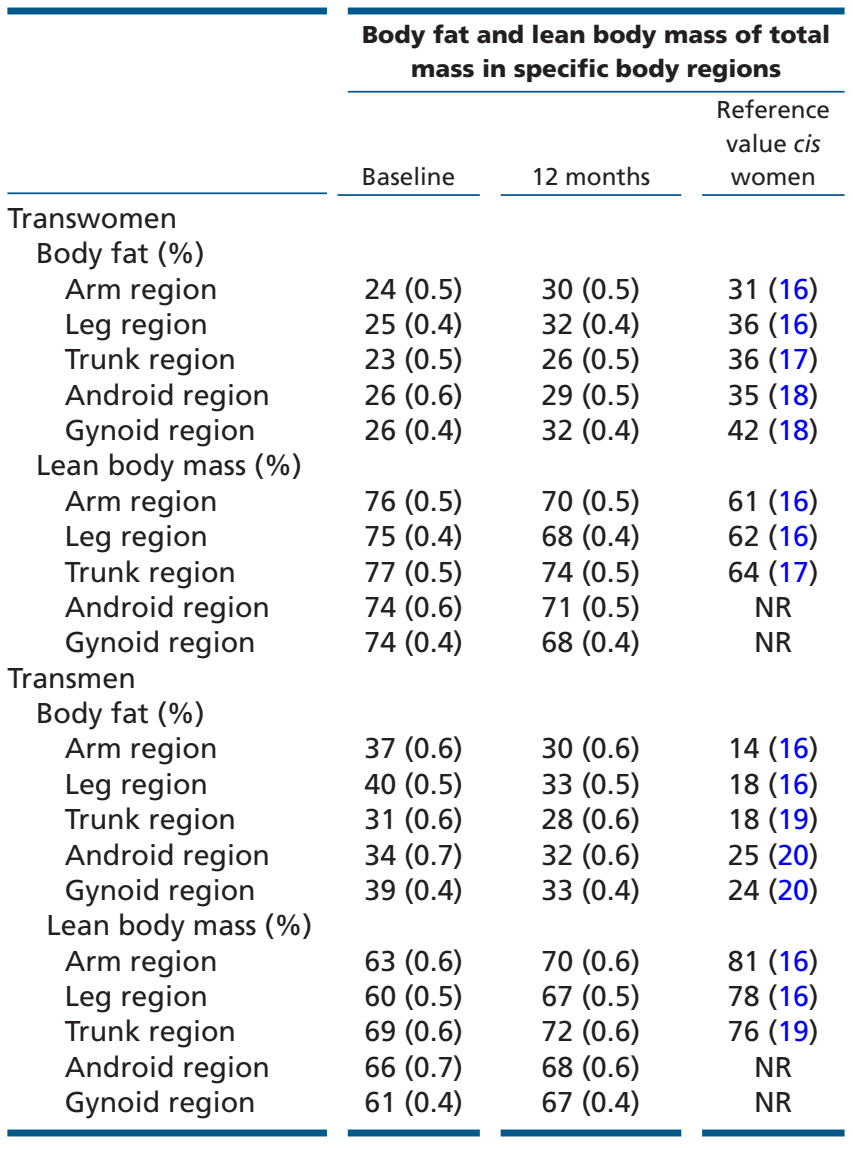

; Age categories for reference values; Women: category 20-29 years old (16), median (range): 31 (18-62) years old (17), mean (s.D.): 20.8 (1.9) years old (18); men: category 20-29 years old (16), median (range): 31

(18-55) years old (19), mean (s.D.): 21.3 (2.2) (20).

$\mathrm{CHT}$, cross-sex hormonal therapy; NR, not reported.

all body fat and lean body mass outcomes. The effects of $\mathrm{T}$ gel and $\mathrm{T}$ undecanoate were similar in most outcome measures. (Fig. 4 and Supplementary Table 1).

\section{Serum estradiol and testosterone level measurements}

In transwomen, no evident associations between mean serum estradiol levels during CHT and total body and regional effects were found on body fat and lean body mass (Supplementary Table 2). In transmen, however, a trend was seen toward a higher decrease in body fat outcomes with increasing $\mathrm{T}$ levels (21)(Supplementary Table 3).

\section{Influence of BMI at start, age and the use of other medication}

A higher BMI at start of CHT was related to smaller increases in body fat and larger decreases in LBM outcomes in transwomen. In transmen, a higher BMI at start was related to larger decreases in body fat and smaller increases in lean body mass outcomes (Supplementary Table 4). Age at start of therapy was, after adjustment for BMI at start of therapy, not related to changes in body fat and lean body mass outcomes (Supplementary Table 5).

The use of other medication such as antidepressants, antipsychotics, corticosteroids and anti-epileptics did not clearly affect the change in total body fat (difference in percentage change in users vs non-users; transwomen: $+3 \%, 95 \%$ CI: $-6 ; 13, P=0.51$, transmen: $+4 \%, 95 \%$ CI: $-2 ; 9, P=0.23$ ), total lean body mass (difference in percentage change in users vs non-users; transwomen $2 \%, 95 \%$ CI: $0 ; 4, P=0.07$, transmen: $-1 \%, 95 \%$ CI: $-3 ; 1$, $P=0.41)$ or regional outcome measures before and after adjustment for age and BMI at start.

\section{Discussion}

This prospective study in 179 transwomen and 162 transmen with measurements before and after 12 months of cross-sex hormone treatment showed that in transwomen the percentage increase in body fat is larger in the gynoid region than in the android region, consistent with a more feminine body fat distribution and a lower WHR. In transmen, percentage body fat in the gynoid region decreased with no change in the android region, consistent with a more masculine body fat distribution and a lower hip circumference. Further, we found that type of hormonal therapy or the concentration of estradiol did not affect changes in regional body fat or lean body mass in transwomen. However, transmen using $\mathrm{T}$ esters seem to experience larger changes in body composition than transmen using $\mathrm{T}$ gel, possibly due to our other finding that transmen with higher $\mathrm{T}$ levels showed larger changes in body fat and lean body mass. In addition, BMI at start appears to be a determinant of changes in body composition. Trans persons with a BMI $<20$ underwent larger changes in all body fat measures (transwomen) or body fat in the android region (transmen) in comparison with their normal-weight (BMI 20-25), overweight (BMI 25-30) and obese (BMI >30) counterparts. Whether these observations are due to their low BMI or to the 
fact that overweight and obese patients were probably keen on losing weight (a BMI of $<30$ is required for sex reassignment surgery in our centers) is unknown.

Our results largely support the results of the few small studies that have been performed so far $(3,4,7)$. A previous study with magnetic resonance imaging examined transverse images of the abdomen, hip and thigh and also showed larger changes in body fat in the gluteofemoral region than in the abdominal region in both transwomen and transmen (3). More recent studies reported similar effects on body fat and lean body mass in the arms and legs with comparable changes in waist and hip circumferences $(4,7)$, although one study reported an increase in waist circumference of 4 centimeters in transwomen (8).

This is the first large prospective observational study in transgender persons presenting the effects of $\mathrm{CHT}$ on regional body composition by objective DXA measures, whereas in previous studies, changes in these regions were only estimated by anthropometry $(7,13)$. Owing to the large sample size, we were able to examine the influence of several important factors on the effects. A limitation of this study is that, because of the multicenter design, different laboratories using different assays were involved, resulting in different serum estradiol and testosterone concentrations. However, we used $Z$-scores to be able to combine the analyses of the two different centers.

Percentage changes in regional body fat and regional lean body mass up to $42 \%$ occurred in the first year of CHT, which all contributed to the feminization or masculinization of separate body parts. Percentage body fat in the arm in transwomen after 12 months of CHT is almost the same as seen in women (Table 2). However, this is not the case in other body parts in which the reference values of the desired sex are approached but not achieved after 12 months of CHT. Possibly, body composition values of the desired sex are achieved after prolonged CHT, but this is yet unknown.

Despite that the same dose of CHT was given to all transwomen, varying estradiol levels were achieved, possibly partly due to a different expression of the estradiol receptor. However, this present study showed that higher serum estradiol levels did not result in larger changes in body fat and lean body mass. This is a relevant finding since in clinical practice, many transwomen wonder whether they would experience larger effects if their hormone dosage would be increased. In transmen, larger effects of $\mathrm{T}$ esters on many outcome measures were observed compared with $\mathrm{T}$ gel and $\mathrm{T}$ undecanoate. However, the use of $\mathrm{T}$ esters involves highly fluctuating testosterone levels, which poorly resemble the male hormonal milieu compared with other testosterone preparations (14). Therefore, on the basis of our results, only it cannot be concluded that $\mathrm{T}$ esters are the optimal treatment option.

This study describes the effects of CHT on body composition in the context of feminization and masculinization by examining objective measures such as regional fat depots and WHR. Future research should focus on whether these effects in trans persons are also reflected in a changing physical appearance and body image. For example, to determine whether the increase in body fat and the decrease in lean body mass in the arm of transwomen causes the arm to look more feminine and to feel softer. Furthermore, studies with long-term follow-up are needed in order to examine whether transwomen and transmen ultimately obtain the body composition of the desired sex. In addition, further research into the effects of CHT on body fat distribution is needed in the context of cardiovascular risk. An excess of intra-abdominal or visceral fat may alter free fat acid metabolism and the release of adipokines and thereby play an important role in the onset of insulin resistance and the metabolic syndrome (15). Therefore, present studies should be extended to assess the effects of $\mathrm{CHT}$ on visceral fat volume and the consequent change in metabolic risk profile in trans persons.

In conclusion, CHT causes a more feminine body fat distribution and a lower WHR in transwomen and a more masculine body fat distribution with a lower hip circumference in transmen. Type of estradiol therapy or the concentration of estradiol does not affect changes in regional body fat or lean body mass in transwomen, while transmen using $\mathrm{T}$ esters experience larger changes in some outcomes than others, possibly due to higher $\mathrm{T}$ levels.

Supplementary data

This is linked to the online version of the paper at https://doi.org/10.1530/ EJE-17-0496.

\section{Declaration of interest}

The authors declare that there is no conflict of interest that could be perceived as prejudicing the impartiality of this study.

\section{Funding}

This research did not receive any specific grant from any funding agency in the public, commercial or not-for-profit sector. 


\section{Acknowledgements}

The authors are grateful to Jos Megens and Kaatje Toye for their contribution to the study.

\section{References}

1 Wells JCK. Sexual dimorphism of body composition. Best Practice and Research Clinical Endocrinology and Metabolism 200721 415-430. (https://doi.org/10.1016/j.beem.2007.04.007)

2 Klaver M, Dekker MJHJ, de Mutsert R, Twisk JWR \& den Heijer M. Cross-sex hormone therapy in transgender persons affects total body weight, body fat and lean body mass: a meta-analysis. Andrologia 201649 e12660. (https://doi.org/10.1111/and.12660)

3 Elbers JHH, Giltay EJ, Teerlink T, Scheffer PG, Asscheman H, Seidell JC \& Gooren LJG. Effects of sex steroids on components of the insulin resistance syndrome in transsexual subjects. Clinical Endocrinology 200358 562-571. (https://doi.org/10.1046/j.13652265.2003.01753.x)

4 van Caenegem E, Wierckx K, Taes Y, Schreiner T, Vandewalle S, Toye K, Lapauw B, Kaufman J-M \& T'Sjoen G. Body composition, bone turnover, and bone mass in trans men during testosterone treatment: 1-year follow-up data from a prospective case-controlled study (ENIGI). European Journal of Endocrinology 2015172 163-171. (https://doi.org/10.1530/eje-14-0586)

5 van de Grift TC, Cohen-Kettenis PT, Steensma TD, De Cuypere G, Richter-Appelt H, Haraldsen IR, Dikmans REG, Cerwenka SC \& Kreukels BPC. Body satisfaction and physical appearance in gender dysphoria. Archives of Sexual Behavior 201645 575-585. (https://doi. org/10.1007/s10508-015-0614-1)

6 Gaber Y. Secondary lymphoedema of the lower leg as an unusual side-effect of a liquid silicone injection in the hips and buttocks. Dermatology 2004208 342-344. (https://doi.org/10.1159/000077845)

7 Wierckx K, van Caenegem E, Schreiner T, Haraldsen IR, Fisher AD, Toye K, Kaufman J-M \& T'Sjoen G. Cross-sex hormone therapy in trans persons is safe and effective at short-time follow-up: results from the european network for the investigation of gender incongruence. Journal of Sexual Medicine 201411 1999-2011. (https:// doi.org/10.1111/jsm.12571)

8 Elbers JMH, Asscheman H, Seidell JC \& Gooren LJG. Effects of sex steroids hormones on regional fat depots as assessed by magnetic resonance imaging in transsexuals. American Journal of Physiology 1999276 317-325.

9 Dekker MJHJ, Wierckx K, van Caenegem E, Klaver M, Kreukels BPC, Elaut E, Fisher AD, van Trotsenburg MAA, Schreiner T, Den Heijer $\mathrm{M}$ et al. A European Network for the investigation of gender incongruence: endocrine part. Journal of Sexual Medicine 201613 994-999. (https://doi.org/10.1016/j.jsxm.2016.03.371)
10 American Psychiatric Association. Diagnostic and Statistical Manual of Mental Disorders, 4th edition, text revision (DSM-IV-TR), Arlington, VA: American Psychiatric Publishing, Inc; 2000.

11 American Psychiatric Association. Diagnostic and Statistical Manual of Mental Disorders, 5th edition. Text revision (DSM-5); Arlington, VA: American Psychiatric Publishing Inc. 2013

12 Hologic Inc. Discovery QDR Series Operator Manual, Hologic Inc, Bedford, MA, USA 2012.

13 Pelusi C, Costantino A, Martelli V, Lambertini M, Bazzocchi A, Ponti F, Battista G, BVenturoli S \& Meriggiola MC. Effects of three different testosterone formulations in female-to-male transsexual persons. Journal of Sexual Medicine 201411 3002-3011. (https://doi. org/10.1111/jsm.12698)

14 Giagulli VA, Triggiani V, Cornona G, Carbone D, Licchelli B, Tafaro E, Resta F, Sabbà C, Maggi M \& Guastamacchia E. Evidencebased medicine update on Testosterone Replacement Therapy (TRT) in male hypogonadism: focus on new formulations. Current Pharmaceutical Design 201117 1500-1511. (https://doi. org/10.2174/138161211796197160)

15 Després JP \& Lemieux I. Abdominal obesity and metabolic syndrome. Nature 2006444 881-887. (https://doi.org/10.1038/nature05488)

16 Coin A, Giannini S, Minicuci N, Rinaldi G, Pedrazzoni M, Minisola S, Rossini M, Del Puente A, Inelmen EM, Manzato E et al. Limb fat-free mass and fat mass reference values by dual-energy X-ray absorptiometry (DEXA) in a 20-80 year-old Italian population. Clinical Nutrition 201231 506-511. (https://doi.org/10.1016/j. clnu.2012.01.012)

17 Bracco D, Thiébaud D, Chioléro RL, Landry M, Burckhardt P \& Schutz Y. Segmental body composition assessed by bioelectrical impedance analysis and DEXA in humans. Journal of Applied Physiology 199681 2580-2587.

18 Stults-Kolehmainen MA, Stanforth PR \& Bartholomew JB. Fat in android, trunk, and peripheral regions varies by ethnicity and race in college aged women. Obesity 201220 660-665. (https://doi. org/10.1038/oby.2011.300)

19 Fuller NJ, Laskey MA \& Elia M. Assessment of the composition of major body regions by dual-energy X-ray absorptiometry (DEXA), with special reference to limb muscle mass. Cinical Physiology 199212 253-266. (https://doi.org/10.1111/j.1475-097X.1992. tb00831.x)

20 Stults-Kolehmainen MA, Stanforth PR, Bartholomew JB, Lu T, Abolt CJ \& Sinha R. DXA estimates of fat in abdominal, trunk and hip regions varies by ethnicity in men. Nutrition and Diabetes 20133 e64. (https://doi.org/10.1038/nutd.2013.5)

21 Bui HN, Sluss PM, Blincko S, Knol DL, Blankenstein MA \& Heijboer AC. Dynamics of serum testosterone during the menstrual cycle evaluated by daily measurements with an ID-LC-MS/MS method and a 2nd generation automated immunoassay. Steroids 2013 78 96-101. (https://doi.org/10.1016/j.steroids.2012.10.010)

Received 22 June 2017

Revised version received 31 October 2017

Accepted 22 November 2017 